\title{
ORIENTAÇÕES DE ENFERMAGEM A PACIENTES PÓS-TRANSPLANTE RENAL: REVISÃO INTEGRATIVA
}

\author{
Nursing guidelines for patients after kidney transplantation: integrative review \\ Camila Trindade Leandro, Katya Araújo Machado Saito, Maryana da Silva Furlan, \\ Vera Lúcia Andrade de Aquino
}

\section{RESUMO}

Introdução: Nos últimos anos, a doença renal crônica (DRC) vem aumentando substancialmente. Com isso, aumentou o número de pacientes na fila de espera para um transplante. Dessa forma, faz-se necessário que os enfermeiros estejam capacitados para prestar assistência aos pacientes desde a fase pré-transplante até a alta hospitalar e o seguimento ambulatorial. Objetivo: Avaliar as evidências disponíveis na literatura sobre orientações de enfermagem para pacientes transplantados renais. Método: Trata-se de uma revisão integrativa da literatura, realizada no período de março a outubro de 2019. A busca e coleta dos dados foram realizadas através do acesso on-line ao Portal CAPES e BVS, utilizando-se descritores em Ciência da Saúde (Decs): Transplante renal, Normas e Enfermagem. Utilizou-se critério de inclusão, artigos na íntegra, na língua portuguesa, inglesa e espanhola, publicados no período de 2014 a 2018. Resultados: Dentre os 344 artigos selecionados, 331 foram eliminados por não atenderem ao objetivo e aos critérios de inclusão; assim, analisaram-se 13 artigos. Conclusão: O estudo mostrou a importância do enfermeiro no cuidado direto na assistência a pacientes transplantados renais, orientando-os quanto à importância do tratamento imunossupressor, aos cuidados com a alimentação e hábitos de vida saudáveis, na intenção de proporcionar maior qualidade de vida e evitando perda do enxerto e até morte do paciente.

Descritores: Transplante Renal; Normas; Enfermagem.

\section{Instituição:}

Programa de Residência Uniprofissional em Enfermagem ClínicoCirúrgica da Sociedade Beneficente de Senhoras Hospital Sírio Libanês, São Paulo/SP - Brasil

\section{Correspondência:}

Camila Trindade Leandro

Av. Pres. João Café Filho, 1331, CEP 09811323, S.Bernardo do Campo/SP (11) $97768-4437$

camiladtrindade@gmail.com

Recebido em: 18/09/2019
Aceito em: $21 / 10 / 2019$

\section{INTRODUÇÃO}

A doença renal crônica (DRC) é uma síndrome resultante de danos renais progressivos e irreversíveis a partir de moléstias prévias, como: hipertensão arterial sistêmica (HAS), Diabetes Mellitus (DM), infecções urinárias de repetição, cálculo renal, nefrites e má formação do aparelho urinário, que se refletem no mal funcionamento renal. ${ }^{1}$

O rim é um órgão que exerce diversas funções no organismo humano, como a excreção de produtos finais de diversos metabolismos que são tóxicos ao corpo, produção de hormônios, controle do equilíbrio hidroeletrolítico, controle do metabolismo ácidobásico e, principalmente, da pressão arterial. A função 
excretora renal pode ser medida através da Taxa de Filtração Glomerular (TFG). ${ }^{1}$

Os pacientes com DRC são classificados em cinco estágios. Há sinais de falência renal quando a DRC se encontra em estágio avançado, estágio 5 , dialítico ou não diádico. ${ }^{2}$ A DRC é caracterizada por apresentar Taxa de Filtração Glomerular (TFG) menor que $60 \mathrm{ml} /$ $\min / 1,73 \mathrm{~m}^{2}$ por pelo menos três meses. ${ }^{1}$

Além da DRC ter vários estágios, ainda é uma doença progressiva e irreversível da função renal, progressão que pode levar muitos desses pacientes para a DRC terminal. Esses pacientes necessitam de algum tipo de terapia renal substitutiva (TRS) como a hemodiálise, a diálise peritoneal e o transplante renal. ${ }^{1}$

O diagnóstico da DRC é bem simples e pode ser realizado através de exames de urina, principalmente para avaliar o valor de albumina, exames de sangue para avaliar o valor de ureia, creatinina e calculando a TFG, e também de exames de imagem, como ultrassonografia de rins e vias urinárias. ${ }^{1}$

A DRC é um problema de saúde pública em todo o mundo e tem como opções de tratamento a HD, diálise peritoneal e o transplante renal no estágio 5. Para isso, faz-se necessário que o paciente apresente boas condições para a cirurgia e para o tratamento póstransplante, com o uso dos imunossupressores. ${ }^{1,2}$

O Sistema Público de Saúde (SUS) tem o maior sistema de transplante público do mundo. Segundo dados da Coordenação Geral do Sistema Nacional de Transplante, o transplante renal corresponde a $25 \%$ do total dos transplantes de órgãos realizados no Brasil. ${ }^{3,4}$

A posição na fila de transplante não é constante, podendo ser alterada devido às prioridades, como crianças e pacientes de maior gravidade, como com falência de acesso vascular, impossibilitando a realização de HD. ${ }^{5,6}$

O transplante renal tem como maior objetivo proporcionar mais qualidade de vida ao paciente e sua reintrodução socioeconômica, com menor custo social. É um procedimento cirúrgico, realizado pela ablação de um rim de um doador vivo ou falecido e posteriormente a implantação desse rim em um receptor. ${ }^{4}$

Esse processo é de extrema importância tanto para o doador quanto para o receptor, pois apesar de ser uma nova experiência para a família, doador e receptor, permeiam aspectos psicossociais, anseios, medos, tristeza e insegurança quanto ao sucesso da cirurgia e como será a vida após o transplante. ${ }^{5}$

Estudos apontam que a experiência dos pacientes transplantados após o procedimento cirúrgico é complexa. O primeiro ano pós-transplante é o período de maior desafio para o enfermeiro orientar o paciente. Portanto, é preciso assegurar que as informações passadas para o paciente e a família sejam realizadas de forma adequada e assertiva, transmitindo segurança e garantindo que o paciente saberá prover o seu próprio cuidado após a cirurgia. ${ }^{6}$

O enfermeiro tem papel imensurável no acolhimento e orientação, com o objetivo de oferecer um serviço de qualidade, humanizado e integral para pacientes transplantados, orientação sobre dieta, cuidados com o enxerto, ferida operatória, cuidados com a tomada dos medicamentos imunossupressores, controle da diurese, atividade física, orientação quanto à atividade sexual, regularidade nas consultas com nefrologista, imunização e a importância da realização dos exames de função renal e taxa de tacrolimus, que em altas doses pode ser nefrotóxico. ${ }^{6,7}$

No período de atuação no cenário prático e vivência em um serviço de transplante durante o estágio optativo, identificou-se a importância do tema devido à grande relevância nas práticas de saúde. Portanto, a questão norteadora deste estudo foi: qual a importância das orientações de Enfermagem realizadas no pósoperatório de transplante renal? E o objetivo: avaliar as evidências disponíveis na literatura sobre as orientações de Enfermagem para pacientes transplantados renais.

\section{MÉTODOS}

Trata-se de uma revisão integrativa da literatura, de abordagem qualitativa, com o objetivo de avaliar as evidências disponíveis na literatura sobre as orientações de Enfermagem para pacientes transplantados renais. A revisão integrativa é baseada na construção de uma análise da literatura, com o objetivo de contribuir na discussão de métodos e resultados, gerando reflexão e possibilitando novos estudos. Seu objetivo principal é proporcionar um conhecimento mais específico acerca de um determinado assunto, a partir de estudos anteriores. ${ }^{8,9}$

É uma metodologia de pesquisa que agrega vários tipos de estudos de diferentes métodos, na intenção de sintetizar os resultados encontrados, agregando novos conhecimentos, ratificando o conhecimento prévio e contribuindo na tomada de decisão e melhorias na prática clínica. ${ }^{8,9}$

$\mathrm{Na}$ pesquisa realizada, utilizou-se a estratégia PICO (Paciente, Intervenção, Comparação, "Outcomes"): o "P" representou os pacientes de transplante renal, o "l" são as orientações de Enfermagem nesse grupo específico, o "C" não se aplica nessa pesquisa e, por fim, 
o "O" significa apresentar os resultados e a importância de orientar os pacientes de transplante renal. Assim, a estratégia PICO possibilita a construção de uma pergunta norteadora adequada, bem estruturada, com boas evidências científicas nas bases de dados, durante a pesquisa. ${ }^{10}$

Desta maneira, para a construção de uma revisão integrativa, faz-se necessário percorrer seis principais etapas: ${ }^{8,10}$

1) O estabelecimento da hipótese ou questão a pesquisa, que nada mais é do que a escolha do tema a ser abordado, sendo esse tema relacionado à prática clínica, delimitar os objetivos e identificar as palavras-chave; $;, 9$

2) Realizar a busca na literatura, estabelecendo os critérios de inclusão e exclusão, a escolha das bases de dados que serão utilizadas e seleção dos estudos que farão parte da pesquisa a ser desenvolvida;

3) A terceira etapa consiste na categorização dos estudos, extraindo as informações relevantes nas pesquisas selecionadas para a formação do banco de dados; ${ }^{8}$

4) Avaliação dos estudos com análise crítica dos mesmos, incluindo ou excluindo artigos para a confecção da revisão integrativa, sendo os principais critérios a análise crítica e o conhecimento do revisor acerca do assunto a ser estudado;
5) Realizar a interpretação dos resultados com discussão dos mesmos, incluindo a avaliação crítica dos estudos e comparação com os conhecimentos teóricos prévios;

6) Baseia-se na elaboração do percurso metodológico, com a descrição das etapas percorridas na pesquisa, com a exposição dos principais resultados encontrados e os artigos incluídos. ${ }^{8}$

Utilizou-se a pesquisa nas seguintes bases de dados: Portal de periódicos CAPES e BVS, que elencaram as estratégias de busca, utilizando os Descritores em Ciências da Saúde - DeCS/MeSH com o termo "descritor de assunto", juntamente com o elemento de combinação AND.

A busca foi realizada a partir das seguintes estratégias e seus respectivos resultados, segundo ano de publicação: (Figura 1).

Utilizou-se como critérios de inclusão: artigos originais escritos na íntegra que tratavam do assunto proposto publicados em português, inglês e espanhol, no período de 2014 a 2018. Critérios de exclusão: artigos nas demais línguas, publicados fora do período estabelecido e que não retratassem o tema proposto. Após leitura em pares (leitura realizada por dois revisores de forma independente) dos títulos dos artigos encontrados a partir dos critérios de inclusão e exclusão e da utilização dos filtros disponíveis nas plataformas de busca, foram

Figura 1: Artigos encontrados, por ano de publicação na BVS e Capes

\begin{tabular}{|c|c|c|c|c|c|c|c|c|c|c|c|}
\hline $\mathbf{N}$ & Estratégias & BVS2014 & BVS2015 & BVS2016 & BVS2017 & BVS2018 & Capes2014 & Capes2015 & Capes2016 & Capes2017 & Capes2018 \\
\hline 01 & (ti:(kidney*)) AND (ti:(communic*)) & 1 & 2 & 1 & 1 & 1 & 320 & 383 & 345 & 568 & 724 \\
\hline 02 & (ti:(Transplant*)) AND (ti:(orient*)) & 1 & 0 & 1 & 2 & 3 & 308 & 405 & 329 & 438 & 580 \\
\hline 03 & (ti:(Transplant*)) AND (ti:(educ*)) & 2 & 2 & 0 & 0 & 1 & 277 & 343 & 291 & 442 & 631 \\
\hline 04 & (ti:(Transplantat*)) AND (ti:(communic*)) & 0 & 2 & 1 & 0 & 1 & 191 & 239 & 197 & 242 & 337 \\
\hline 05 & (ti:(Transplantat*)) AND (ti:(educ*)) & 5 & 4 & 6 & 11 & 8 & 12 & 15 & 14 & 11 & 26 \\
\hline 06 & (ti:(Transplant*)) AND (ti:(vida)) & 5 & 3 & 6 & 13 & 5 & 6 & 6 & 10 & 5 & 8 \\
\hline 07 & (ti:(Transplant*)) AND (ti:(cuida*)) & 4 & 5 & 5 & 0 & 3 & 525 & 670 & 547 & 777 & 1058 \\
\hline 08 & (ti:(Transplant*)) AND (ti:(experien*)) & 1 & 7 & 3 & 1 & 1 & 164 & 206 & 171 & 270 & 379 \\
\hline 09 & (ti:(Transplant*)) AND (ti:(viv*)) & 5 & 2 & 4 & 7 & 8 & 483 & 616 & 555 & 773 & 1010 \\
\hline 10 & (ti:(Transplantat*)) AND (ti:(care*)) & 14 & 8 & 10 & 22 & 7 & 968 & 1147 & 1046 & 1633 & 1959 \\
\hline 11 & (ti:(kidney*)) AND (ti:(care*)) & 1 & 2 & 1 & 1 & 1 & 320 & 383 & 345 & 568 & 724 \\
\hline 12 & (ti:(renal $\left.\left.{ }^{*}\right)\right)$ AND (ti:(communic $\left.\left.c^{*}\right)\right)$ & 1 & 0 & 1 & 0 & 1 & 232 & 297 & 263 & 384 & 548 \\
\hline 13 & (ti:(renal*)) AND (ti:(guida*)) & 1 & 0 & 1 & 1 & 0 & 162 & 216 & 213 & 281 & 373 \\
\hline 14 & (ti:(renal*)) AND (ti: (care*)) & 4 & 10 & 4 & 4 & 2 & 839 & 1025 & 917 & 1333 & 1738 \\
\hline 15 & $\left(\mathrm{ti}:\left(\mathrm{renal}^{*}\right)\right)$ AND (ti:(life $\left.\left.{ }^{*}\right)\right)$ & 10 & 12 & 7 & 9 & 5 & 900 & 1041 & 943 & 1405 & 1801 \\
\hline 16 & (ti:(kidney*)) AND (ti:(life $\left.\left.{ }^{*}\right)\right)$ & 24 & 17 & 18 & 6 & 9 & 1127 & 1349 & 1272 & 1965 & 2399 \\
\hline 17 & $\left(\mathrm{ti}:\left(\mathrm{renal}^{*}\right)\right)$ AND $\left(\mathrm{ti}:\left(\mathrm{educ}^{\star}\right)\right)$ & 1 & 1 & 0 & 0 & 2 & 362 & 417 & 387 & 493 & 614 \\
\hline 18 & (ti:(renal*)) AND (ti:(nurs $\left.\left.{ }^{\star}\right)\right)$ & 0 & 1 & 0 & 1 & 0 & 162 & 209 & 188 & 258 & 343 \\
\hline 19 & $\left(\mathrm{ti}:\left(\operatorname{discharg}^{*}\right)\right)$ AND $\left(\mathrm{ti}:\left(\operatorname{transplant}{ }^{\star}\right)\right)$ & 4 & 2 & 4 & 0 & 0 & 115 & 162 & 149 & 217 & 267 \\
\hline
\end{tabular}


selecionados 274 artigos da BVS e 70 da CAPES, totalizando 344 artigos selecionados.

Posteriormente, após a leitura em pares dos resumos dos artigos selecionados e utilizando os critérios de inclusão e exclusão, a amostra final desta revisão integrativa foi constituída de oito artigos da BVS e cinco da CAPES.

Para a análise e posterior síntese dos artigos que atenderam aos critérios de inclusão, foi utilizado um quadro sinóptico com as seguintes informações: título, autores, ano de publicação, tipo de pesquisa e objetivos, principais resultados e conclusões. Através desse roteiro, foi realizada uma análise confrontando as opiniões distintas dos autores e agrupando ideias semelhantes dos mesmos. Por se tratar de revisão integrativa da literatura, o presente estudo não foi submetido a um comitê de ética.

\section{RESULTADOS E DISCUSSÃO}

$\mathrm{Na}$ presente revisão integrativa, analisaram-se treze artigos que atenderam aos critérios de inclusão previamente estabelecidos e a seguir, apresentou-se um panorama geral dos artigos selecionados e avaliados. (Figura 2)

Figura 2: Número total de artigos encontrados/selecionados/ utilizados

\begin{tabular}{|l|ll|c|c|}
\hline Ano & \multicolumn{2}{|l|}{ Artigos encontrados } & $\begin{array}{l}\text { Artigos } \\
\text { selecionados }\end{array}$ & $\begin{array}{l}\text { Artigos } \\
\text { utilizados }\end{array}$ \\
\hline 2014 & $7.501(14,4 \%)$ & 81 & 3 \\
\hline 2015 & $9.107(17,5 \%)$ & 74 & 1 \\
\hline 2016 & $8.167(15,7 \%)$ & 75 & 2 \\
\hline 2017 & $11.901(22,9 \%)$ & 60 & 2 \\
\hline 2018 & $15.268(29,4 \%)$ & 54 & 5 \\
\hline Total & 51.944 & 344 & 13 \\
\hline
\end{tabular}

A partir dos resultados encontrados no período de 2014 a 2018 com a utilização das estratégias de busca, o ano de 2018 apresentou o maior número de artigos publicados $(29,4 \%)$, enquanto que o ano de 2014 apresentou menor número $(14,4 \%)$.

Dentre os 344 artigos selecionados, 331 foram eliminados por não atenderem ao objetivo e critérios de inclusão; assim, foram analisados 13 artigos, que estão descritos na Figura 3.

Observou-se que nos treze artigos analisados, dois estão relacionados à qualidade de vida dos pacientes de transplante renal, quatro relacionados à educação e orientação do paciente pré-transplante renal, dois sobre o diagnóstico de Enfermagem, um sobre cuidados do paciente com o enxerto, um sobre qualidade da assistência de Enfermagem, dois sobre cuidados de Enfermagem e um sobre orientações de alta hospitalar. Isso demostra que as orientações de Enfermagem e a educação do paciente transplantado ainda são prioridades e, após a educação, vem a qualidade de vida como reflexo dos cuidados de Enfermagem para esses pacientes.

É principalmente de competência de o enfermeiro fazer orientações referentes à alta ao paciente transplantado, à importância do acompanhamento ambulatorial, ao uso correto dos imunossupressores (tacrolimus e myfortic) e dos exames de rotina (ureia, creatinina, eletrólitos, taxa de tacrolimus), hábitos alimentares, ingesta hídrica, atividade física e, principalmente, cuidados com higiene. ${ }^{11}$

Partindo desse pressuposto, a ferramenta de comunicação, relação de vínculo e confiança transmitida pelo enfermeiro são essenciais para que se tenha uma orientação eficaz. Através da comunicação, o enfermeiro cria uma conexão com o paciente e seus familiares, permitindo, assim, que se estabeleça um entendimento maior e mais preciso das orientações de Enfermagem, assegurando melhor qualidade no autocuidado. ${ }^{11}$

Isso ocorre também pelo fato de o enfermeiro ser o profissional na linha de frente dos cuidados, tendo maior tempo na assistência ao paciente. Assim, assegurando a informação e orientações passadas ao paciente, fazse necessário que o mesmo repita e reafirme o que foi orientado pelo enfermeiro e demais profissionais na assistência a esse paciente e esclareça todas as suas dúvidas, ainda durante o período de internação. ${ }^{12}$

O transplante é um procedimento muito delicado e requer muitos cuidados após a sua realização. Faz-se necessário que o paciente tenha conhecimento dos efeitos colaterais e principalmente, dos riscos aos quais ele está exposto, para que possa realizar o autocuidado com o menor risco possível.

Os principais efeitos adversos da terapia imunossupressora são: risco de infecções, neoplasias, obesidade, DM, HAS e nefrotoxicidade, podendo levar a internações recorrentes. $^{12}$

As orientações de Enfermagem são de imensurável importância aos pacientes pós-transplante renal no momento da alta hospitalar, focando nas orientações dos cuidados e na atenção ao tomar as medicações, na dose e hora correta, para evitar a rejeição do enxerto e auxiliar no bom funcionamento do órgão. ${ }^{12}$ 
Figura 3: Distribuição dos artigos segundo título, autores, ano de publicação, objetivos e resultado

\begin{tabular}{|c|c|c|c|c|}
\hline Título & Autores & Ano & Objetivos & Resultados \\
\hline $\begin{array}{l}\text { Qualidade de vida } \\
\text { pós-transplante renal: } \\
\text { revisão integrativa }\end{array}$ & $\begin{array}{l}\text { Santos RP, } \\
\text { Rocha DLB }\end{array}$ & 2014 & $\begin{array}{l}\text { Buscar evidências disponíveis na literatura } \\
\text { sobre a melhoria na qualidade de vida de } \\
\text { pacientes após o transplante renal. }\end{array}$ & $\begin{array}{l}\text { Finalmente, considera-se necessário que a equipe } \\
\text { de Enfermagem desempenhe o papel de educação } \\
\text { em saúde junto aos pacientes, cuidadores e } \\
\text { familiares, ainda no pré-operatório, preparando- } \\
\text { os e informando-os sobre suas responsabilidades, } \\
\text { bem como esclarecendo quaisquer dúvidas. }\end{array}$ \\
\hline $\begin{array}{l}\text { Qualidade de Vida em } \\
\text { Transplantados Renais }\end{array}$ & $\begin{array}{l}\text { Santos LF, } \\
\text { Prado BC, } \\
\text { Castro FPS, } \\
\text { Brito RF, } \\
\text { Maciel SC, } \\
\text { Avelar TC }\end{array}$ & 2018 & $\begin{array}{l}\text { traçar o perfil sócio demográfico, } \\
\text { compreender o significado do transplante } \\
\text { renal e seu impacto na qualidade de vida. } \\
\text { Participaram } 12 \text { pacientes transplantados, } \\
\text { em acompanhamento ambulatorial, em } \\
\text { um hospital público, localizado em Recife }\end{array}$ & $\begin{array}{l}\text { O transplante favorece a melhoria da qualidade } \\
\text { de vida dos pacientes, além disso, pode nortear } \\
\text { os profissionais de saúde e os pacientes que } \\
\text { vislumbram a realização desse procedimento. }\end{array}$ \\
\hline $\begin{array}{l}\text { Comunicação no } \\
\text { relacionamento } \\
\text { interpessoal } \\
\text { Enfermeiro/paciente } \\
\text { com indicação de } \\
\text { transplante renal }\end{array}$ & $\begin{array}{l}\text { Oliveira AM, } \\
\text { Soares E }\end{array}$ & 2016 & $\begin{array}{l}\text { Comunicação entre enfermeiro e paciente } \\
\text { em processo hemodialítico, com vistas ao } \\
\text { transplante, verificar o direcionamento } \\
\text { do paciente ao procedimento cirúrgico, } \\
\text { averiguar as orientações realizadas no } \\
\text { pré-operatório mediato e discutir a } \\
\text { importância da comunicação enfermeiro/ } \\
\text { paciente }\end{array}$ & $\begin{array}{l}\text { Portanto, ainda é necessário repensar o papel do } \\
\text { enfermeiro como educador, esclarecendo, mas } \\
\text { também na interpretação do que o indivíduo } \\
\text { quer, adaptando seu trabalho à realidade para a } \\
\text { rotina da prática de Enfermagem, aconselhando o } \\
\text { paciente com entusiasmo ao autocuidado. }\end{array}$ \\
\hline $\begin{array}{l}\text { The Health-Care } \\
\text { Provider's Perspective } \\
\text { of Education Before } \\
\text { Kidney Transplantation }\end{array}$ & $\begin{array}{l}\text { Trivedi P, } \\
\text { Rosaasen N, } \\
\text { Mansell H }\end{array}$ & 2016 & $\begin{array}{l}\text { Identificar déficits em conhecimento } \\
\text { do paciente na perspectiva de uma } \\
\text { equipe multidisciplinar de transplante e } \\
\text { determinar se suas percepções alinham-se } \\
\text { com pacientes que já foram submetidos a } \\
\text { um transplante }\end{array}$ & $\begin{array}{l}\text { Apesar da educação pré e pós-transplante, } \\
\text { surgiram temas específicos, incluindo concepções } \\
\text { errôneas sobre o processo de avaliação e a lista } \\
\text { de espera e a cirurgia, a incongruência entre } \\
\text { as expectativas do paciente e o resultado e a } \\
\text { confusão em relação aos medicamentos. As } \\
\text { percepções dos profissionais de saúde foram } \\
\text { notavelmente consistentes com os receptores de } \\
\text { transplantes. }\end{array}$ \\
\hline $\begin{array}{l}\text { A Comunicação } \\
\text { como Ferramenta } \\
\text { Educativa no Pré- } \\
\text { Operatório Mediato de } \\
\text { Transplante Renal }\end{array}$ & $\begin{array}{l}\text { Oliveira AM, } \\
\text { Soares E }\end{array}$ & 2018 & $\begin{array}{l}\text { Reconhecer a importância da comunicação } \\
\text { do enfermeiro, como ferramenta utilizada } \\
\text { durante o período pré-operatório de } \\
\text { pacientes submetidos à terapia de } \\
\text { hemodiálise e indicado para transplante } \\
\text { renal. }\end{array}$ & $\begin{array}{l}\text { Salientando a relevância do estudo do processo } \\
\text { de comunicação, porque, colocando-o em } \\
\text { prática, há melhoria em relação às habilidades de } \\
\text { comunicação do enfermeiro e, evitando qualquer } \\
\text { interferência que possa afetar as informações } \\
\text { fornecidas. }\end{array}$ \\
\hline $\begin{array}{l}\text { Implementation of a } \\
\text { new patient education } \\
\text { programme for Renal } \\
\text { transplant recipients }\end{array}$ & $\begin{array}{l}\text { Urstad KH, } \\
\text { Wahl AK, } \\
\text { Engebretsen } \\
\text { E, Larsen MH, } \\
\text { Vidnes TK, } \\
\text { Stenwig AGK, } \\
\text { Simensen } \\
\text { OW, Nordli A, } \\
\text { Reisæter AV, } \\
\text { Andersen MH }\end{array}$ & 2018 & $\begin{array}{l}\text { Investigar a satisfação, a competência, o } \\
\text { treinamento e a percepção da qualidade } \\
\text { da assistência no trabalho de Enfermagem } \\
\text { em relação à implementação de um novo } \\
\text { programa de educação para o paciente, } \\
\text { baseado em evidências }\end{array}$ & $\begin{array}{l}\text { O novo programa foi encontrado para ser mais } \\
\text { estruturado, centrado no paciente e visível para } \\
\text { os enfermeiros em todas as alas, em comparação } \\
\text { com a sua prática anterior. Enfermeiros com } \\
\text { menor experiência em Enfermagem foram } \\
\text { significativamente mais motivados sobre a nova } \\
\text { educação do paciente pelo programa, do que os } \\
\text { enfermeiros mais experientes. }\end{array}$ \\
\hline $\begin{array}{l}\text { Nursing Diagnoses } \\
\text { Among Kidney } \\
\text { Transplant Recipients: } \\
\text { Evidence From Clinical } \\
\text { Practice }\end{array}$ & $\begin{array}{l}\text { Lukasewicz } \\
\text { AS, Ferreira } \\
\text { RN, Echer } \\
\text { ICRN, Lucena } \\
\text { AF }\end{array}$ & 2014 & $\begin{array}{l}\text { Identificar os diagnósticos de Enfermagem } \\
\text { aplicados aos receptores de transplante } \\
\text { renal em um hospital brasileiro. }\end{array}$ & $\begin{array}{l}\text { Seis diagnósticos de Enfermagem foram mais } \\
\text { prevalentes entre os receptores de transplante de } \\
\text { rim no período pós-operatório: risco de infecção, } \\
\text { eliminação urinária prejudicada, proteção ineficaz, } \\
\text { déficit no auto-cuidado: banho, Integridade tissular } \\
\text { prejudicada e dor aguda. }\end{array}$ \\
\hline $\begin{array}{l}\text { Frecuencia de los } \\
\text { diagnósticos de } \\
\text { enfermería en el } \\
\text { primer ingreso del } \\
\text { paciente con un } \\
\text { trasplante renal } \\
\text { reciente }\end{array}$ & $\begin{array}{l}\text { Cabana AF, } \\
\text { Rebé MI, } \\
\text { Rodríguez } \\
\text { MR, Polonio } \\
\text { CE, Andrea } \\
\text { TL, Jiménez } \\
\text { DM }\end{array}$ & 2017 & $\begin{array}{l}\text { Descrever os principais DxE na primeira } \\
\text { admissão do paciente após transplante } \\
\text { renal recente. }\end{array}$ & $\begin{array}{l}\text { Conhecendo o NxD mais frequente, permite } \\
\text { estabelecer planos de cuidado, com qualidade, } \\
\text { e tentam prevenir possíveis complicações do } \\
\text { transplante que afetem a qualidade de vida do } \\
\text { paciente. }\end{array}$ \\
\hline
\end{tabular}




\begin{tabular}{|c|c|c|c|c|}
\hline Título & Autores & Ano & Objetivos & Resultados \\
\hline $\begin{array}{l}\text { Cuidados realizados } \\
\text { pelas pessoas com } \\
\text { transplante renal para } \\
\text { a manutenção do } \\
\text { órgão }\end{array}$ & $\begin{array}{l}\text { Santos BP, } \\
\text { Lise F, Feijó } \\
\text { AM, } \\
\text { Garcia RP, } \\
\text { Schwartz E }\end{array}$ & 2017 & $\begin{array}{l}\text { Identificar os cuidados com o transplante } \\
\text { renal, realizados pelas pessoas, para a } \\
\text { manutenção do órgão transplantado }\end{array}$ & $\begin{array}{l}\text { Os principais cuidados realizados pelas pessoas } \\
\text { com o transplante renal, para a manutenção } \\
\text { do órgão transplantado estão relacionados à } \\
\text { alimentação, ingesta hídrica, higiene, às atividades } \\
\text { sociais e laborais, medicações e à saúde. }\end{array}$ \\
\hline $\begin{array}{l}\text { Perspectives } \\
\text { on Quality of } \\
\text { Care in Kidney } \\
\text { Transplantation: A } \\
\text { Semistructured } \\
\text { Interview Study }\end{array}$ & $\begin{array}{l}\text { Brett KE, } \\
\text { Ertel E, } \\
\text { Grimshaw J, } \\
\text { Knoll GA }\end{array}$ & 2018 & $\begin{array}{l}\text { Verificar as opiniões de pacientes, médicos } \\
\text { e administradores de programas sobre } \\
\text { cuidados de qualidade para pacientes } \\
\text { transplantados renais. }\end{array}$ & $\begin{array}{l}\text { Foram identificados } 8 \text { temas: acesso ao } \\
\text { tratamento; acessibilidade dos serviços recursos } \\
\text { do programa; comunicação de informaçães; } \\
\text { atitude dos prestadores de cuidados; desfechos em } \\
\text { saúde; satisfação do paciente e segurança. }\end{array}$ \\
\hline $\begin{array}{l}\text { Percepções de } \\
\text { enfermeiros e clientes } \\
\text { sobre cuidados de } \\
\text { Enfermagem no } \\
\text { transplante de rim }\end{array}$ & $\begin{array}{l}\text { Santos CM, } \\
\text { Kirchmaier } \\
\text { FM } \\
\text { Silveira WJ, } \\
\text { Sena CA }\end{array}$ & 2015 & $\begin{array}{l}\text { Analisar as percepções de enfermeiros } \\
\text { e dos transplantados sobre a consulta } \\
\text { de Enfermagem pré-transplante do } \\
\text { transplante renal. }\end{array}$ & $\begin{array}{l}\text { A consulta de Enfermagem no período pré- } \\
\text { transplante renal é importante para a incorporação } \\
\text { das orientações às vivências e comportamentos } \\
\text { das pessoas transplantadas, ao longo do processo } \\
\text { de transplantação e após a realização do } \\
\text { procedimento. }\end{array}$ \\
\hline $\begin{array}{l}\text { Relação dialógica } \\
\text { com o cliente sobre } \\
\text { transplante renal: } \\
\text { cuidado educativo de } \\
\text { Enfermagem }\end{array}$ & $\begin{array}{l}\text { Ferreira } \\
\text { SAMN, } \\
\text { Teixeira MLO, } \\
\text { Branco EMSC }\end{array}$ & 2018 & $\begin{array}{l}\text { Descrever os saberes de clientes renais } \\
\text { crônicos sobre o transplante renal } \\
\text { e discutir as contribuições desses } \\
\text { saberes com os cuidados educativos de } \\
\text { Enfermagem. }\end{array}$ & $\begin{array}{l}\text { Nesse contexto, o papel do enfermeiro transcende } \\
\text { o ato de orientar para favorecer a conscientização } \\
\text { das pessoas, ou seja, a percepção que elas têm } \\
\text { de si, a respeito da situação em que vivem e das } \\
\text { consequências de suas escolhas para a saúde, } \\
\text { tornando-as sujeitos autônomos na relação do } \\
\text { cuidar. }\end{array}$ \\
\hline $\begin{array}{l}\text { Atuação do enfermeiro } \\
\text { nas orientações de } \\
\text { alta ao paciente pós- } \\
\text { transplante Renal }\end{array}$ & $\begin{array}{l}\text { Inácio LA, } \\
\text { Montezeli JH, } \\
\text { Sade PMC, } \\
\text { Caveião C, } \\
\text { Hey A }\end{array}$ & 2014 & $\begin{array}{l}\text { Descrever as orientações de alta, pelo } \\
\text { enfermeiro, ao paciente pós-transplante } \\
\text { renal }\end{array}$ & $\begin{array}{l}\text { A primeira categoria descreve quais são as } \\
\text { orientações fornecidas pelo enfermeiro ao } \\
\text { paciente no momento da alta. Na segunda } \\
\text { categoria, são abordados aspectos da } \\
\text { comunicação, como competência fundamental a } \\
\text { esse profissional para o momento das orientações } \\
\text { de alta e, na terceira, discorre acerca do } \\
\text { conhecimento científico como alicerce para as } \\
\text { orientações de alta pós-transplante renal. }\end{array}$ \\
\hline
\end{tabular}

Além disso, faz-se necessária a comunicação da importância da dieta pobre em sal e o aumento da ingesta hídrica, o que era diferente antes do transplante. Após o transplante, o paciente deve ingerir pelo menos de dois a três litros de água por dia. Verificar os sinais vitais, conferir o peso diariamente e realizar atividade física conforme solicitação médica são orientações que ajudam na recuperação e no bom funcionamento do organismo.

Todas as orientações devem ser dadas na forma mais clara e objetiva possível, para que o paciente e seus familiares não tenham dúvidas após a alta hospitalar. Para isso, faz-se necessário um plano de alta hospitalar e principalmente, que o enfermeiro tenha conhecimento técnico-científico para poder orientar o paciente. ${ }^{12,13}$

Desta maneira, é preciso a colaboração do paciente e dos familiares no compromisso com o cuidado e atenção nas orientações de Enfermagem, evitando que haja um agravamento do quadro, levando à hospitalização, perda do enxerto e até à morte do paciente. O plano de cuidado de Enfermagem é essencial para o auxílio na orientação dos cuidados para com os pacientes transplantados, tendo em vista a valorização das necessidades do paciente, diagnósticos de Enfermagem, planejamento dos cuidados, execução e implementação dos cuidados. ${ }^{13}$

A qualidade de vida é um fator primordial quando se escolhe a terapia de transplante em pacientes de DRC, com perspectiva de melhora de todos os segmentos da vida do paciente. Isso implica diretamente na saúde física, nas relações sociais, e nas relações familiares, sendo esta a mais importante. Estudos apontam que o controle emocional e psicológico influencia diretamente no transplante, devendo-se levar em consideração os medos e anseios dos pacientes, pois a recuperação e a qualidade de vida devem ser retomadas após o transplante, requerendo, assim, muitos cuidados e entrega dos pacientes e familiares. ${ }^{14,15}$ 
Assim que o transplante ocorre e o enxerto recobra suas funções com êxito, sabe-se que o uso dos imunossupressores é o maior desafio, tanto para a equipe multiprofissional quanto para o paciente, pois seus efeitos colaterais e a necessidade de tomar essas medicações interferem no seu cotidiano; no entanto, eles têm a consciência de que esse tratamento é a única solução para o sucesso do transplante, evitando o regresso à hemodiálise.

Observa-se que, apesar da disponibilidade dos imunossupressores nos postos do SUS, ainda existem dificuldades socioculturais que impossibilitam a adesão desses medicamentos pelos pacientes. Além das limitações vividas por eles, ainda existe receio das reações colaterais dos imunossupressores e do risco de infecções. A Enfermagem tem o poder e domínio de orientar e acompanhar esses transplantados no momento da alta e ambulatorialmente, incentivando e alertando sobre a importância do uso desses medicamentos, para que o paciente tenha melhor qualidade de vida.

Entende-se que o transplante renal não trará a vida de antes da DRC, mas proporcionará algo muito semelhante ao normal, com o paciente podendo desfrutar mais tempo junto com seus familiares e entes queridos. ${ }^{16,17}$

Após análise crítica dos artigos, foi identificada a falta de publicações científicas, o que é de extrema relevância para a sociedade, devido ao aumento dos casos de DRC e suas complicações.

\section{CONCLUSÃO}

Apesar da literatura disponível, constatou-se que há a necessidade de que mais estudos sejam desenvolvidos sobre essa temática, a qual é de extrema relevância para a saúde da pessoa com transplante renal. O estudo mostrou a importância do enfermeiro no cuidado direto da assistência aos pacientes transplantados renais, orientando-os quanto à importância do tratamento imunossupressor, cuidados com a alimentação e hábitos de vida saudáveis, na intenção de proporcionar maior qualidade de vida, evitando a perda do enxerto e até a morte do paciente.

Percebe-se que ainda há a necessidade de maior intervenção, planos de cuidados e elaboração de material educativo para consolidar as orientações de Enfermagem aos pacientes de transplante renal, focando na importância do uso adequado das medicações, reintrodução do paciente ao seu meio, rotinas de vida, educação alimentar, atividade física, exames de rotina e cuidados a serem tomados com o enxerto.

O estudo mostrou a participação da família nas orientações de Enfermagem para o posterior cuidado com esse paciente. O apoio, não só nas atividades diárias, como na recuperação social e emocional de quem é transplantado, auxilia na reintrodução mais rápida e efetiva às rotinas do paciente, melhorando assim sua qualidade de vida.

Existem lacunas a serem exploradas sobre o papel do enfermeiro nas orientações do paciente após o transplante renal. Os achados deste estudo permitem afirmar que o processo educativo desenvolvido pelo enfermeiro nas orientações de alta do paciente transplantado é significativo para a adesão aos cuidados necessários.

\section{ABSTRACT}

Introduction: In recent years, chronic kidney disease (CKD) has been substantially increasing. With it, the number of patients on the waiting list for a transplant has increased. Thus, it is necessary that nurses are trained to provide assistance to patients from the pre-transplant phase through hospital discharge and outpatient follow-up. Purpose: To assess evidence available in the literature on nursing guidelines for kidney transplant patients. Methods: This is an integrative literature review carried out from March to October 2019. Search and collection of data was performed by online access to the CAPES and VHL Portal using descriptors in Health Science (Decs): Kidney transplantation, standards, nursing. Inclusion criteria used were: articles in full, in Portuguese, English and Spanish, published from 2014 to 2018. Results: Among 344 articles selected, 331 were eliminated for not following the purpose and inclusion criteria, and 13 articles were analyzed. Conclusion: The study showed the importance in the direct care of nurses in assisting kidney transplant patients, guiding them as to the importance of immunosuppressive treatment, food care, healthy lifestyle habits in order to provide a better quality of life and avoiding graft loss and even the death of patients.

Keywords: Kidney transplantation; Standards; Nursing. 


\section{REFERÊNCIAS}

1- Ministério da Saúde, Secretaria de Atenção à Saúde, Departamento de Atenção Especializada e Temática. Diretrizes Clínicas para o Cuidado ao paciente com Doença Renal Crônica - DRC no Sistema Único de Saúde. Brasília: Ministério da Saúde. 2014; p.: 37 p.: il

2- Oliveira AM, Soares E. Comunicação no relacionamento interpessoal enfermeiro/paciente com indicação de transplante renal. Cienc Cuid Saude. 2016 Out/Dez; 15(4):647-54

3-Santos BP, Lise F, Feijó AM, Garcia RP, Schwartz E. Cuidados realizados pelas pessoas com transplante renal para a manutenção do órgão. Recife. Rev Enferm UFPE on line. 2017 ago; 11(8):3108-21

4-Santos CM, Kirchmaier FM, Silveira WJ, Sena CA. Percepções de enfermeiros e clientes sobre cuidados de Enfermagem no transplante de rim. Acta Paul Enferm. 2015;28(4):337-43

5- Oliveira A, Soares E. A Comunicação como Ferramenta Educativa no Pré- Operatório Mediato de Transplante Renal. J. res.: fundam. care. Online. 2018 jul./set; 10(3):753-7

6-Santos LF, Prado BC, Castro FPS, Brito RF, Maciel SC, Avelar TC. Qualidade de Vida em Transplantados Renais. Bragança Paulista. 2018 jan./mar;23(1):163-72

7- Kristin HU, Astrid KW, Eivind E, Marie HL, Tone KV, Anne GK. Implementation of a new patient education programme for renal transplant recipients. Journal of Renal Care. 2018;44(2):106-14

8- Mendes KDS, Silveira RCCP, Galvão CM. Revisão integrativa: método de pesquisa para a incorporação de evidências na saúde e na Enfermagem, Texto Contexto. 2008 Out-Dez;17(4):758-64
9- Soares CB, Hoga LAK, Peduzzi M, et.al. Revisão integrativa: conceitos e métodos utilizados na Enfermagem, Rev Esc Enferm USP. 2014;48(2):335-45

10- Santos CMC, Pimenta CAM, Nobre MRC. A estratégia pico para a construção da pergunta de pesquisa e busca de evidências. Rev Latino-am Enfermagem. 2007 maiojunho;15(3)

11- Ferreira SAMN, Teixeira MLO, Branco EMSC. Relação dialógica com o cliente sobre transplante renal: cuidado educativo de Enfermagem. Cogitare Enferm. 2018; (23)2: e52217

12- Inácio LA, Montezeli JH, Sade PMC, Caveião C, Hey AP. Atuação do enfermeiro nas orientações de alta ao paciente pós-transplante renal. Rev Enferm UFSM. 2014 Abr/ Jun;4(2):323-31

13- Cabana AF, Rebé MI, Rodríguez MR, Polonio CE, Andrea TL, Jiménez DM. Frecuencia de los diagnósticos de enfermería en el primer ingreso del paciente con un trasplante renal reciente. Enferm Nefrol. 2017 enero-marzo; 20(1):76/81

14- Ferreira SAL, Echer IC, Lucena AF. Nursing Diagnoses Among Kidney Transplant Recipients: Evidence From Clinical Practice. International Journal of Nursing Knowledge. 2014;25 (1)

15- Santos RP, Daniele Pontifi LBR. Qualidade de vida postransplante renal. Paraná: Enferm Nefrol. 2014 EneroMarzo;17(1): 51/58

16- Trivedi P, Rosaasen N, Mansell $\mathrm{H}$. The Health-Care Provider's Perspective of Education Before Kidney Transplantation. Prog Transplant. 2016;26(4):322-7

17- Kendra EB, Ertel E, Grimshaw J, AKG. Perspectives on Quality of Care in Kidney Transplantation: A Semistructured Interview Study. Transplantation DIRECT. 2018 Aug 21;4(9):e383 